\title{
Completeness of neutron-, photo-induced and spontaneous fission yields data
}

\author{
B. Pritychenko ${ }^{1, *}, O$. Schwerer $^{2}, J$. Totans $^{1}, V$. Zerkin $^{3}$, and $O$. Gritzay $^{2}$ \\ ${ }^{1}$ National Nuclear Data Center, Brookhaven National Laboratory, Upton, NY 11973-5000, USA \\ ${ }^{2}$ Under contract with National Nuclear Data Center, Brookhaven National Laboratory, Upton, NY 11973-5000, USA \\ ${ }^{3}$ Nuclear Data Section, International Atomic Energy Agency, Wagramer Str. 5, 1220 Wien, Austria
}

\begin{abstract}
Nuclear data collection, evaluation and dissemination activities have been performed worldwide for many years. They are absolutely essential for the overall progress of science and technology to create the complete collections of experimental data sets and associated publications, and store these data in publicly accessible databases. Due to many historical and technological reasons not all published data have been identified and compiled. These "missing data" manifest themselves via scientific publications, data evaluations and nuclear databases comparisons. The detailed analysis of the Nuclear Science References (NSR) and the Experimental Nuclear Reaction (EXFOR) databases shows thousands of previously missed nuclear reaction experiments and creates a roadmap for the creation of complete data records for fission cross sections, yields and covariances. The National Nuclear Data Center (NNDC) program for identification, compilation and storage of missing fission yields data sets is described, and recommendations for improving the databases completeness are given.
\end{abstract}

\section{Introduction}

Compilation and evaluation of neutron cross sections have been pioneered at Brookhaven National Laboratory (BNL) in the early 1950s [1]. The BNL group summarized its findings in the now famous BNL-325 report [2] and developed the SCISRS (Sigma Center Information Storage and Retrieval System) setup [3] for data compilations. Several currently run nuclear reaction data projects such as the Atlas of Neutron Resonances reference book [4], Evaluated Nuclear Data File (ENDF) [5] and Experimental Nuclear Reaction Data (EXFOR) ([6] and references therein) libraries originate from the Brookhaven endeavor.

These efforts laid a foundation for worldwide nuclear data compilation and evaluation activities in subsequent years, and led to the creation of other data centers in Vienna, Austria (Nuclear Data Section or NDS-IAEA), Paris, France (Nuclear Energy Agency or NEA-Databank) and Obninsk, USSR (Center Jadernykh Dannykh or CJD) in 1963-1964. Around 1970 the four neutron data centers agreed on the data interchange format (EXFOR), and later formed the Nuclear Reaction Data Centres (NRDC) network under the auspices of the International Atomic Energy Agency (IAEA) to manage the data compilations worldwide. Responsibilities for reaction data compilations were distributed among the centers on a geographical basis: Area \#1: U.S. and Canada (NNDC), Area \#2 is Western Europe + Japan (NEA-Databank), Area \#3 is Eastern Europe, Africa, Asia, Australia, Latin America (NDSIAEA) and Area \#4: Former USSR (CJD).

*e-mail: pritychenko@bnl.gov

\section{EXFOR Contributions Worldwide}

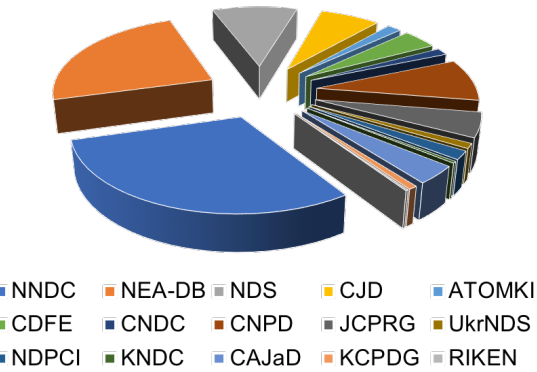

Figure 1. EXFOR database contents include contributions of the currently operating NNDC Brookhaven, NEA-DB Paris, NDS IAEA Vienna, CJD Obninsk, ATOMKI Debrecen, CDFE Moscow, CNDC Beijing, CNPD Sarov, JCPRG Sapporo, UkrNDS Kyiv, NDPCI BARC/India, KNDC Taejon and discontinued CAJaD, KCPDG and RIKEN data centers. Centers descriptions and complementary details are available from the EXFOR compilation control system: https://wwwnds.iaea.org/public/exfor/x4compil/.

The geographical separation of compilation work has been a very productive mechanism for interactions between researchers and data compilers who speak the same language, and it helped to make EXFOR one of the first truly international collaborations. This partnership has produced the only continuouslyupdated low- and intermediate-energy nuclear reaction database that is publicly available from the NDS-IAEA 
or NNDC web sites: https://www-nds.iaea.org/exfor/ or https://www.nndc.bnl.gov/exfor/, respectively. Global contributions to the international EXFOR database are shown in Fig. 1. The Figure data show that NNDC (Area \#1) has provided the largest individual contribution to the EXFOR library followed by NEA-Databank (Area \#2), NDS-IAEA (Area \#3) and others.

\section{Missing Data}

The EXFOR library and its computer database are primary repositary of experimental neutron, charged-particle, and photonuclear reaction data compilations. The development of library contents was determined by historical and technological reasons. The Fig. 2 data show that the initial compilation scope was focused on neutron cross section data, and many important nuclear physics quantities such neutron fission yields, charged-particle and photonuclear reaction parameters were excluded from the compilation scope until 80s. In the early years, the data were either directly obtained from authors or extracted from tables. Unfortunately, due to short comings of computer technologies in the last century not all plotted data sets were recovered from the researchers and many publications were missed. This led to the situation when several important publications were unnoticed by the EXFOR compilers, and the "missing data" problem was created [6]. These missing in EXFOR references can be easily extracted from the NSR database, and their estimate is shown in Table 1 . The large number of potentially missing charged-particle references is due to the EXFOR compilation scope restriction in the 70s. It is worth to notice that multiple publications that originate from the same experiment are grouped in EXFOR into single entry while NSR treats these references as separate entries. The NSR database is the best source for missing nuclear reaction references because Web search engines are not very prolific with old publications. However, it has a few limitations:

- NSR search is based on its keywords, and search brings no results when the reaction keywords are missing

- During the first twenty years of operation at the Oak Ridge National Laboratory (ORNL) NSR was mostly a nuclear structure database [8]

Table 1. Estimate of NSR nuclear reaction references that are missing in EXFOR as of December 2018.

\begin{tabular}{l|c}
\hline \hline Projectile & \# of References \\
\hline $\mathrm{n}$ & 1199 \\
$\mathrm{p}$ & 5994 \\
$\mathrm{~d}$ & 2828 \\
$\mathrm{t}$ & 522 \\
${ }^{3} \mathrm{He}$ & 2167 \\
$\alpha$ & 3042 \\
$\gamma$ & 793 \\
\hline \hline
\end{tabular}

Presently, compilation of nuclear data is a very broad activity that includes nuclear reaction, structure and bibliographic databases or EXFOR, eXperimental Unevaluated

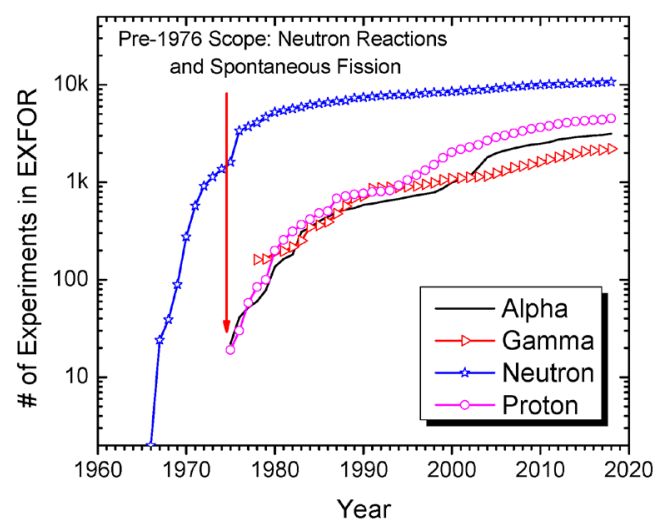

Figure 2. EXFOR historic timeline shows evolution of the library data compilation scope over the years.

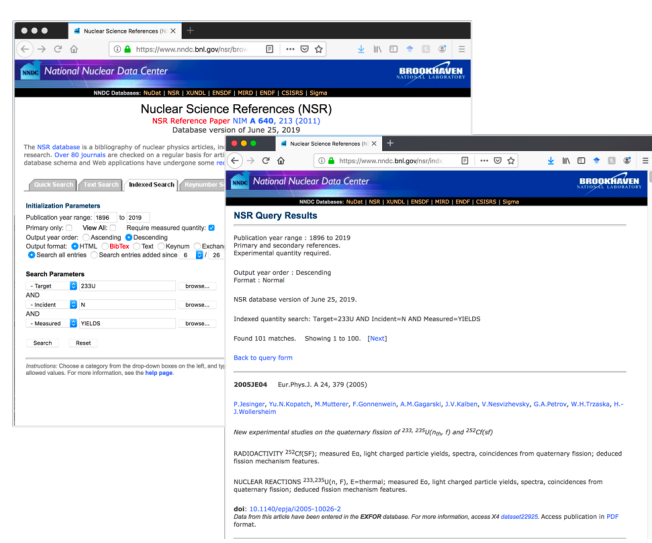

Figure 3. Results of NSR library, https://www.nndc.bnl.gov/nsr, indexed search for the missing in EXFOR ${ }^{233} \mathrm{U}(\mathrm{n}, \mathrm{F})$ fission yields data. The following search parameters $233 \mathrm{U}, \mathrm{N}$ and yields were used for target, incident and measured indexed quantities, respectively.

Nuclear Data List (XUNDL) [7] and Nuclear Science References (NSR) [8], respectively. These databases contain extensive records of nuclear science measurements and are completely based on modern computer technologies. It represents an interest to explore them for fission yields and create complete data records.

In this contribution we would explore the EXFOR and NSR databases as of February of 2018 for missing data. They contained 22294 and 229594 nuclear reaction and bibliographical data entries, respectively. The data search was conducted using the NSR Web Interface (https://www.nndc.bnl.gov/nsr/) that is integrated with the EXFOR database. The NSR database was queried for missing references using the NSR indexed and text searches as shown in Fig.3. The searches for missing neutron-induced (NFY), photo-induced (PFY) and spontaneous fissions (SFY) yields data included measured and deduced quantities to accommodate different NSR compilation styles that changed over the years. 


\section{Analysis of Fission Yields}

The analysis of EXFOR database contents for neutroninduced, spontaneous and photo-induced fission yields showed that it includes 817 experiments, 1611 reactions and 2992 data sets. The Nuclear Science References database has been created as a nuclear structure and decay bibliographical database in 1960, and it may contain many complementary fission yield references. The initial analysis of the NSR database for missing EXFOR data produced 384 neutron-induced, 142 spontaneous and 126 photo-induced fission yields references as shown in Fig. 4; 540 fission yields PDF files were identified and added to the joint EXFOR-NSR PDF database during the analysis.

The completeness check of the EXFOR database against the NSR bibliography for experimental fission product yield data has been described in the NRDC memo CP-C/469 [9], and it consisted of several steps. First, an NSR retrieval for references on fission product yields was made, and references obviously already compiled in EXFOR were excluded, including articles which appear in EXFOR as secondary references to a certain experimental work. Then, references were excluded which do not contain original experimental information, such as evaluations or when fission yield data from the literature were used to obtain other data. It was found that many of the remaining references, though not yet appearing in EXFOR, do belong to existing EXFOR entries, e.g. conference papers or progress reports containing preliminary data. For such references, addition to an existing entry as secondary reference was initiated; in some cases, where an abstract does not contain any information of interest to the users, it is sufficient to keep the reference on record for the data centers without adding it to EXFOR. To assess whether or not an article refers to a work already compiled in EXFOR, the existing EXFOR entries within a time window of \pm 4 years were checked for similar data measured by the same authors at the same laboratory. After this procedure, for the remaining "new" articles the NSR-style bibliographic information (journal titles, laboratory report codes, conference paper identification) was converted to conform to EXFOR coding rules, and the resulting list was summarized in three NRDC memos [10-12] and distributed to the NRDC centers for action. The analysis of missing data in Tables 1 - 2 shows that the NSR estimate is reasonable. The accurate accounting for missing info on B, C, N, O, $\mathrm{F}$ and Ne projectiles, spontaneous fission and the different NSR database scope and keywording style at ORNL would raise the projected size of the EXFOR library from the current $\sim 22,000$ to approximately $\sim 40,000$ experimental works.

In order to estimate the volume of work, the pilot fission yields project was conducted at the National Nuclear Data Center in 2018. The project scope included compilation of 15 new and revision of 18 existing EXFOR fission yield compilations. The compilation time for new and revised entries was 180 and 90 hours, respectively. Several rules for the compilation of fission yields, spallation reactions and beta-shielded nuclei were re-examined during this exercise, and it helped to clarify the possible chal-
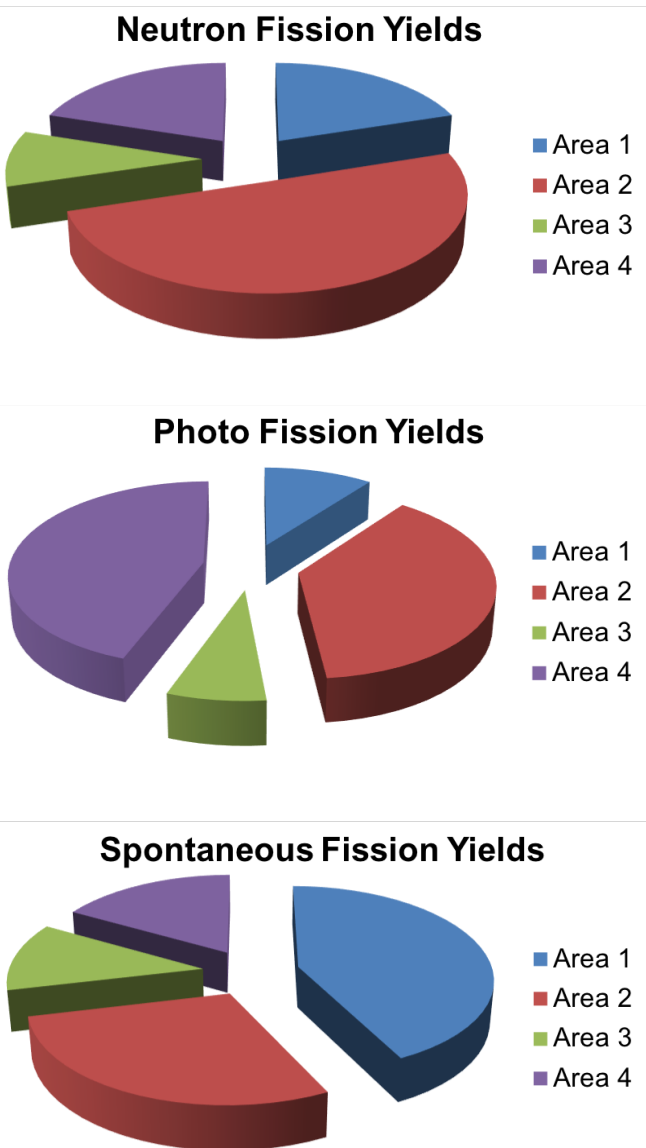

Figure 4. Initial results for geographical distribution of missing neutron-induced, photo-induced and spontaneous fission yields.

lenges of future work. These findings have been used to estimate the workload for the Area \#1 fission yields compilations [10-12] that are now in progress, and first data entries have been submitted to the EXFOR database in Spring 2019. The advanced EXFOR editor [13] is used in this work, and the expected time of completion for the Area \#1 compilations is Fall 2020 while work in other compilation centers may progress at a slower pace.

Table 2. Final results for missing neutron-induced (NFY), photo-induced (PFY) and spontaneous (SFY) fission data sets. Data are taken from the NRDC memos [10-12].

\begin{tabular}{l|c|c|c}
\hline \hline Area/Yields & NFY & PFY & SFY \\
\hline$\# 1$ & 33 & 8 & 35 \\
$\# 2$ & 124 & 32 & 28 \\
$\# 3$ & 26 & 7 & 12 \\
$\# 4$ & 49 & 40 & 25 \\
\hline Total & 232 & 87 & 100 \\
\hline \hline
\end{tabular}

Previously, the issue of completeness of the EXFOR fission yields data was raised in the IAEA coordinated research project [14], and the NRDC network worked on database improvements. Presently, the IAEA is conducting a bibliographical check of England and Rider eval- 
uation [15], and Ph.D. Thesis of Mills [16] for missing references in the EXFOR database [17]. The results of this effort would provide a complementary to the current project information that would help to improve the completeness of EXFOR database. Recent U.S. Department of Energy Nuclear Data Interagency Working Group / Research Program [18] includes fission yields, and the fission yields compilation work would represent a starting point for a new fission yields evaluation between Brookhaven and Los Alamos National Laboratories in collaboration with international partners.

\section{Conclusion}

The current work represents the first comprehensive effort to address the missing data problem for fission yields. The NSR database was extensively searched for missing references, these data were analyzed, the research facilities were identified, PDF files for the articles were collected, and all these materials were passed to the responsible compilation centers. This effort could serve a model for future NRDC activities on missing data.

The NSR-EXFOR database comparison demonstrated the large potential of the two databases and produced multiple complementary findings. These results would create a basis for reevaluation of neutron fission yields that would produce a broad impact in many areas of applied and fundamental science.

The authors are indebted to A. Sonzogni for encouragement of this project, T. Kawano for useful comments, R. Arcilla for a careful reading of the manuscript and valuable suggestions, and we are grateful to the NRDC network members for the continuous work on EXFOR data compilations and overall database improvements. Work at Brookhaven was funded by the Office of Nuclear Physics, Office of Science of the U.S. Department of Energy, under Contract No. DE-AC02-98CH10886 with Brookhaven Science Associates, LLC.

\section{References}

[1] D.J. Hughes, T.W. Bonner, H. Goldstein, W.W. Havens, Jr., I. Kaplan, C.O. Muehlhause, A.H.Snell, J.R. Stehn, T.M. Shyder, R.F. Taschek, A. Wattenberg, "A Compilation of the AEC Neutron Cross Section Advisory Group," Brookhaven National Laboratory RePORT 170, May 15 (1952).

[2] D.J. Hughes, J.A. Harvey, "Neutron Cross Sections," BrookhaVen National Laboratory Report 325, July 1 (1955).

[3] N. Holden, "A Short History of CSISRS: At the Cutting Edge of Nuclear Data Information Storage and Retrieval Systems and its Relationship to CINDA,
EXFOR and ENDF," December 2005. Downloaded from 〈https://www.nndc.bnl.gov/exfor/compilations/ CSISRSHistory.pdf $\rangle$ on June 26, 2019.

[4] S.F. Mughabghab, Atlas of Neutron Resonances, Resonance Properties and Thermal Cross Sections $\mathrm{Z}=1-60$ 1, Elsevier Publisher, Amsterdam (2018).

[5] D.A. Brown, M.B. Chadwick, R. Capote, et al., Nucl. Data Sheets 148, 1 (2018).

[6] V.V. Zerkin, B. Pritychenko, "The experimental nuclear reaction data (EXFOR): Extended computer database and Web retrieval system," Nucl. Instrum. Meth. Phys. Res. A 888, 31 (2018).

[7] eXperimental Unevaluated Nuclear Data List, Downloaded from $\langle$ https://www.nndc.bnl.gov/xundl $\rangle$ on June 26, 2019.

[8] B. Pritychenko, E. Betak, M.A. Kellett, B. Singh, J. Totans, "The Nuclear Science References (NSR) database and Web Retrieval System," Nucl. Instr. Meth. Phys. Res. A 640, 213 (2011).

[9] O. Schwerer, "Procedure used for the completeness check EXFOR vs. NSR on fission yields," NRDC Memo CP-C/469, NDS-IAEA, 14 April 2019.

[10] O. Schwerer, B. Pritychenko, "Completeness check EXFOR vs. NSR: Spontaneous Fission Yields," NRDC Memo CP-C/464, NDS-IAEA, 29 November 2018.

[11] O. Schwerer, B. Pritychenko, "Completeness check EXFOR vs. NSR: Photofission Yields," NRDC Memo CP-C/465, NDS-IAEA, 30 January 2019.

[12] O. Schwerer, B. Pritychenko, "Completeness check EXFOR vs. NSR: Neutron-induced fission yields," NRDC Memo CP-C/466, NDS-IAEA, 19 February 2019.

[13] G.N. Pikulina, S.M. Taova, "Activities of the RFNCVNIIEF Center of Nuclear Physics Data on the Compilation of Experimental Data for the EXFOR International Library: EXFOR-EDITOR Software Package," Phys.Atomic Nuclei 81, 1450 (2018).

[14] H.O. Denschlag, T.R. England, A.A. Goverdovski, et al., "Compilation and evaluation of fission yield nuclear data, Final report of a co-ordinated research project 1991-1996," International Atomic Energy Agency Report IAEA-TECDOC-1168, December 2000.

[15] T.R. England, B.F. Rider, "Evaluation and Compilation of Fission Product Yields 1993," Los Alamos National Laboratory Report LA-UR-94-3106 (1994).

[16] R.W. Mills, "Fission product yield evaluation," Thesis, Univ. of Birmingham (1995).

[17] S. Okumura et al., ND 2019 Proceedings (2020).

[18] "Nuclear Data Interagency Working Group / Research Program," DOE National Laboratory Announcement Number: LAB 18-1903. Downloaded from 〈https://www.energy.gov/science/np/nuclearphysics $\rangle$ on June 26, 2019. 